Document downloaded from:

http://hdl.handle.net/10251/150627

This paper must be cited as:

Nowacka, AE.; Briantais, P.; Prestipino, C.; Llabrés I Xamena, FX. (2019). Selective Aerobic Oxidation of Cumene to Cumene Hydroperoxide over Mono- and Bimetallic Trimesate Metal Organic Frameworks Prepared by a Facile Green Aqueous Synthesis. ACS Sustainable Chemistry \& Engineering. 7(8):7708-7715. https://doi.org/10.1021/acssuschemeng.8b06472

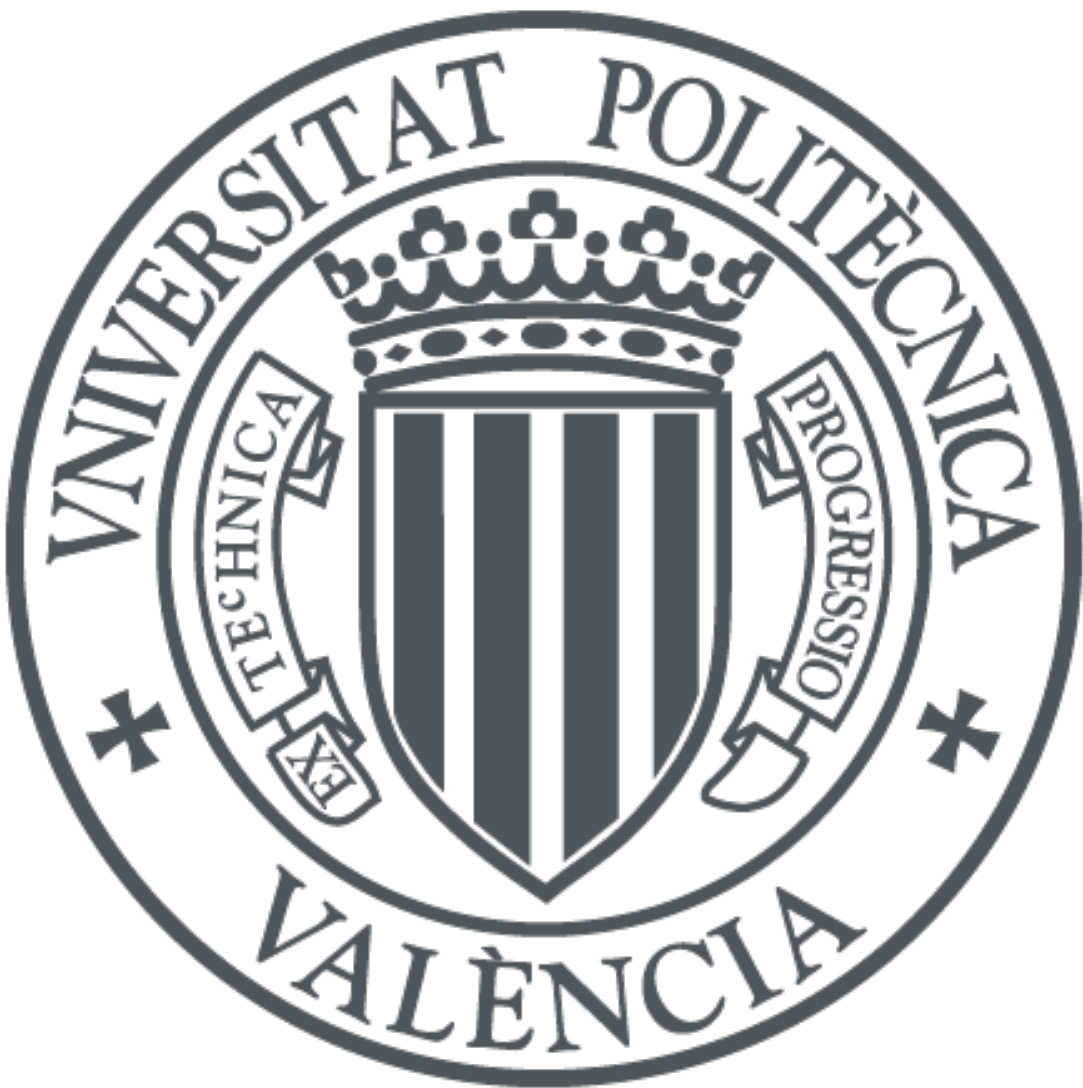

The final publication is available at

https://doi.org/10.1021/acssuschemeng.8b06472

Copyright American Chemical Society

Additional Information

"This document is the Accepted Manuscript version of a Published Work that appeared in final form in ACS Sustainable Chemistry \& Engineering, copyright (C American Chemical Society after peer review and technical editing by the publisher. To access the final edited and published work see https://doi.org/10.1021/acssuschemeng.8b06472" 


\title{
Selective aerobic oxidation of cumene to cumene hydroperoxide over mono- and bimetallic trimesate metal-organic frameworks prepared by a facile "green" aqueous synthesis
}

\author{
Anna Nowacka, ${ }^{a}$ Pol Briantais, ${ }^{b}$ Carmelo Prestipino ${ }^{b, *}$ and Francesc X. Llabrés i \\ Xamena ${ }^{a, *}$ \\ ${ }^{a}$ Instituto de Tecnología Química, Universitat Politècnica de València, Consejo Superior \\ de Investigaciones Científicas, Avda. de los Naranjos, s/n, 46022 Valencia, Spain \\ ${ }^{b}$ Univ Rennes, CNRS, ISCR (Institut des Sciences Chimiques de Rennes) - UMR 6226, \\ 35000 Rennes, France
}

* Corresponding authors: fllabres@itq.upv.es

carmelo.prestipino@univ-rennes1.fr

\begin{abstract}
:
Co-Ni and Mn-Ni bimetallic trimesate MOFs prepared by a fast aqueous synthesis method are excellent and reusable catalysts for the selective oxidation of cumene to cumene hydroperoxide. Isolation of $\mathrm{Co}^{2+}\left(\right.$ or $\left.\mathrm{Mn}^{2+}\right)$ in an inert Ni-BTC framework is a good strategy to optimize CHP selectivity above $90 \%$ : since only $\mathrm{Co}^{2+}$ sites catalyze CHP decomposition, a drop of the CHP selectivity is observed as the cobalt content increases. The statistical probability of having isolated $\mathrm{Co}^{2+}$ sites is calculated as a function of the total cobalt content of the bimetallic compound, assuming homogeneous distribution of $\mathrm{Co}^{2+}$ ions in the Ni-BTC framework and preferential occupation of terminal sites. Thus, in our best sample, with a $\mathrm{Co}: \mathrm{Ni}$ ratio of $5: 95,73 \%$ of the total $\mathrm{Co}^{2+}$ ions are isolated so that CHP decomposition/over-oxidation processes at the surface of the catalyst are not likely to occur before CHP desorption. This can explain the excellent CHP selectivity (91\%) attained over this material. This "site isolation" effect is further supported by similar findings on Mn-Ni bimetallic compounds.
\end{abstract}

Keywords : MOF catalysis - Aerobic cumene oxidation - Bimetallic MOFs - Site isolation $\cdot$ Single-site catalysts 


\section{INTRODUCTION}

Selective aerobic oxidation of cumene (CM) to cumene hydroperoxide (CHP) (Scheme 1) is a relevant reaction from the industrial point of view, since CHP is an important intermediate in the production of phenol, which can then be further converted into phenolic resins and polycarbonates via bisphenol A. ${ }^{1-2}$ Near $90 \%$ of phenol produced worldwide is obtained through the cumene route. CHP is also used in the Sumitomo process as oxygen carrier for the production of propylene oxide..$^{3-4}$<smiles>CC(C)c1ccccc1</smiles>

$\mathrm{CM}$

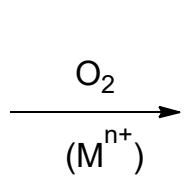<smiles>CC(C)(O)c1ccccc1</smiles>

$\mathrm{CHP}$

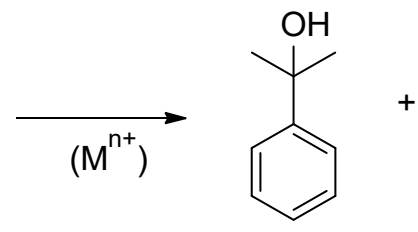

$\mathrm{PP}$<smiles>CC(=O)c1ccccc1</smiles>

AP

Scheme 1. Aerobic oxidation of cumene (CM) to cumene hydroperoxide (CHP). The first reaction can be auto-catalytic or catalyzed by metal ions $\left(\mathrm{M}^{\mathrm{n}+}\right)$. These $\mathrm{M}^{\mathrm{n}+}$ ions can also catalyze CHP decomposition (second reaction), leading to the formation of 2-phenyl-2propanol (PP) and acetophenone (AP) as the main by-products.

Typically, CM oxidation to CHP is carried out in the absence of any catalyst (autooxidation process) and using small amounts of self-initiator CHP, 2,2'-Azobis(2methylpropionitrile) (AIBN) or $N$-hydroxyphthalimide $(\mathrm{NHPI})^{5-8}$ in the temperature range between $80^{\circ} \mathrm{C}$ and $120^{\circ} \mathrm{C}$ and from atmospheric pressure up to 7 bars in air. Main side products of the reaction are 2-phenyl-2-propanol (PP) and acetophenone (AP). As all organic peroxides, CHP is sensitive to heat, and to the presence of acids, metal ions etc. For this reason, alkaline agents like $\mathrm{NaOH},{ }^{9} \mathrm{Na}_{2} \mathrm{CO}_{3},{ }^{10}$ or $\mathrm{NH}_{4} \mathrm{NaCO}_{3},{ }^{11}$ are commonly added to neutralize the formation of organic acid by-products and prevent phenol formation, which can have negative effects on selectivity and productivity of CHP. ${ }^{12}$ Selectivity to CHP attained under these conditions is typically above 90-92\%. However, the productivity of such autooxidation processes (expressed in terms of grams of CHP produced per liter of $\mathrm{CM}$ and per hour; $\mathrm{g} \mathrm{L}^{-1} \mathrm{~h}^{-1}$ ) is usually below the desired values. Therefore, in order to increase the CHP productivity, while maintaining the excellent selectivity to CHP, different catalysts have been proposed, either homogeneous or heterogeneous, mainly based on transition metal salts and oxides. ${ }^{13-21}$ 
We and others have shown that several Metal-Organic frameworks (MOFs) can be used for the aerobic oxidation (i.e., using only air or oxygen as the oxidant) of activated alkanes, such as tetralin, ${ }^{22}$ cyclooctane,${ }^{23}$ indane, ${ }^{24}$ and various alkylbenzenes, ${ }^{25}$ including cumene. Alternatively, MOFs can also be used as hosts in which other active species, such as metallo-phthalocyanines ${ }^{26}$ or metal-oxide nanoparticles. ${ }^{27}$

In spite of their high potential, catalyst preparation is an important limitation for the large scale application of MOFs. Indeed, they are usually prepared in DMF or other organic solvents that can be toxic and/or dangerous to handle, and often require the use of non-commercial and/or expensive organic ligands, which introduce additional synthesis steps and rise up the final price of the catalyst, making the resulting materials less economically appealing, as compared to cheaper alternative catalysts. Moreover, the use of high synthesis temperatures $\left(100-200^{\circ} \mathrm{C}\right.$ in many cases), overpressures, and long synthesis times (one week or longer in certain cases) are also undesired characteristics of most existing MOF preparations described so far. ${ }^{28-31}$

In a precedent report, we described the preparation of various isoreticular mono$\left(\mathrm{Co}^{2+}, \mathrm{Ni}^{2+}, \mathrm{Cu}^{2+}\right.$ and $\left.\mathrm{Zn}^{2+}\right)$ and bimetallic (Co-Ni, $\left.\mathrm{Co}-\mathrm{Zn}, \mathrm{Mn}-\mathrm{Ni}\right)$ trimesate MOFs using a facile and "green" synthesis method. ${ }^{32}$ (This manuscript is provided to the reviewers as a "Supporting Information for review only") The procedure used showed a series of advantages that improved the sustainability and industrial feasibility of the MOF preparation, including: synthesis from non-toxic, aqueous solutions, room temperature and atmosphere pressure, very short synthesis time ( $\sim 10 \mathrm{~min}$.), high yield ( $>90 \%)$, easy separation and washing protocols, easily scalable method, and a cheap and commercially available ligand precursor, trimesic acid $\left(\mathrm{H}_{3} \mathrm{BTC}\right)$. Herein, we extended our studies on "green" MOFs by exploring their catalytic properties as selective catalysts for the aerobic liquid phase oxidation of cumene to cumene hydroperoxide.

\section{EXPERIMENTAL SECTION}

Materials preparation. The synthesis method used for preparing mono- and bimetallic trimesate MOFs was described in detail elsewhere. ${ }^{32}$ Briefly, an aqueous solution of trisodium benzene-1,3,5-tricarboxylate $\left(\mathrm{Na}_{3} \mathrm{BTC}\right)$ was poured onto a suitable divalent metal salt solid precursor at room temperature under vigorous stirring. The amounts of $\mathrm{Na}_{3} \mathrm{BTC}$ solution and metal precursor used were adjusted to have a 2:3 molar ratio. Ethanol (1:2 v:v ratio with respect to total water) was also added to the above mixture, to accelerate the precipitation of the MOF and to increase the final yield obtained, which 
was typically higher than $90 \%$ in all cases. Stirring of the mixture was continued for 10 minutes, and the solid obtained was separated by centrifugation and carefully washed with distilled water (three times) and ethanol (once). Finally, the powder so obtained was air-dried overnight at room temperature. The materials will be hereafter referred to as either M-BTC or MxM'-BTC, for monometallic or bimetallic compounds, respectively. $\mathrm{M}$ and $\mathrm{M}$ ' indicate the metal(s) used in each case, while $\mathrm{x}$ represents the (nominal) molar percentage of metal ions $\mathrm{M}$ in the bimetallic compound.

Catalytic studies. CAUTION!! The following reaction conditions have been carefully selected to avoid flammable mixtures. Any deviation from these conditions must be carefully evaluated. In a typical catalytic experiment, $1 \mathrm{~mL}(7.14 \mathrm{mmol})$ of cumene (CM, 99\% Sigma-aldrich) and the desired amount of solid catalyst were placed inside a homemade glass microreactor (volume $=6 \mathrm{~mL}$ ) equipped with magnetic stirring, a manometer, a gas inlet and a liquid sampling valve. The reactor was connected with a cannula to an $\mathrm{O}_{2}$ supply system, fixed at a total pressure of 4 bars. This reservoir system provides a continuous supply to restock the $\mathrm{O}_{2}$ consumed during the oxidation reaction, so that the concentration of $\mathrm{O}_{2}$ was constant throughout the reaction. The reactors were placed in iron hot-plate at the desired temperature. Time-evolution of products was carried out by GC analysis (Agilent Technologies 7890A, capillary column $10 \mathrm{~m} \times 320 \mu \mathrm{m} \times 0.1 \mu \mathrm{m}$ ) on sample aliquots taken at fixed time intervals. The samples were injected directly oncolumn to minimize the possible decomposition of the hydroperoxide at the injector.

\section{RESULTS AND DISCUSSION}

Structural characterization of mono- and bimetallic trimesates. In a previous paper we described in detail the synthesis and structural characterization of the MOFs studied herein, so we will only briefly summarize the main conclusions extracted from our previous study. ${ }^{32}$

The aqueous synthesis method used produced highly crystalline, isoreticular Co, $\mathrm{Ni}, \mathrm{Cu}$ and $\mathrm{Zn}$-BTC monometallic compounds of general formula $\mathrm{M}_{3} \mathrm{BTC}_{2} \cdot 12 \mathrm{H}_{2} \mathrm{O}$, analogous to those first synthetized by Yaghi and co-workers using a conventional solvothermal method. ${ }^{33}$ These compounds feature a layered structure consisting of infinite zigzag chains aligned along [101] direction that are formed by the alternation of BTC with $\mathrm{M}$ atoms. The repeating unit (black rectangle in Fig. 1) contains two types of $\mathrm{M}$ atoms, bridging and terminal, in a 2:1 ratio. Bridging $\mathrm{M}$ atoms are connected to two BTC units in unidentate mode along the chain, while terminal $\mathrm{M}$ atoms are coordinated 
in a bidentate fashion by one free carboxylate group on alternate BTC molecules. Individual zigzag chains stack along the $b$ axis (Fig. 1b) to form layers in the (10-1) plane, while these layers are stacked along the $c$ axis with an ABA arrangement (Fig. 1c and d).

a)

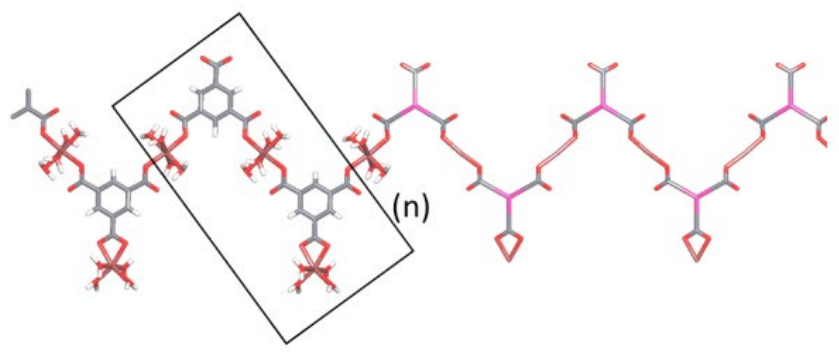

c)

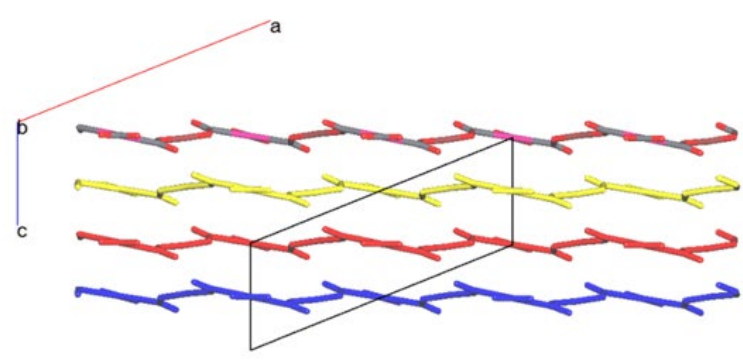

b)

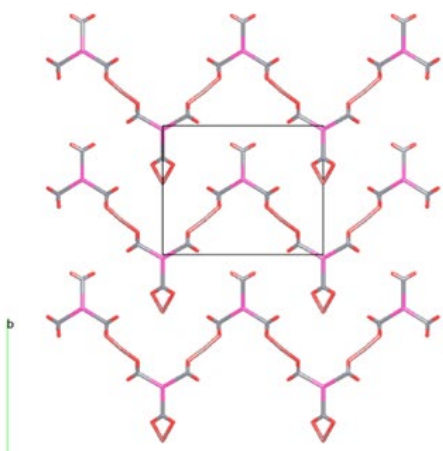

d)

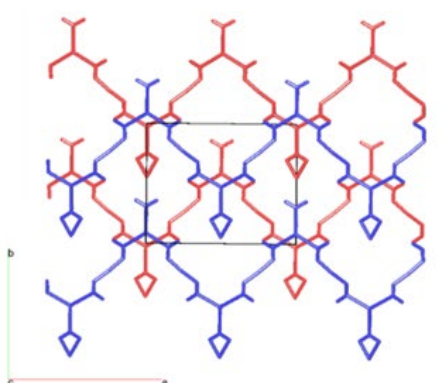

Figure 1. a) $\mathrm{M}_{3} \mathrm{BTC}_{2} \cdot 12 \mathrm{H}_{2} \mathrm{O}$ zigzag chains. The black rectangle single out the repeating unit. b) Single $\mathrm{M}_{3} \mathrm{BTC}_{2} \cdot 12 \mathrm{H}_{2} \mathrm{O}$ layer representation along (001) direction. Hydrogen atoms and water molecules have been removed for clarity. Cell borders are indicated by black lines. Layer packing representations along (001) and (010) directions are shown in parts c) and d) respectively. Chain are colored depending from the layer in which they lay.

By adapting the above method leading to monometallic trimesates, we succeeded in preparing isoreticular $\mathrm{Co}-\mathrm{Ni}$ and $\mathrm{Co}-\mathrm{Zn}$ binary mixtures in the entire range of compositions (i.e., from $0 \%$ to $100 \%$ of cobalt in each series), as well as $\mathrm{Mn}-\mathrm{Ni}$ compounds up to a maximum Mn concentration of 50\%. By combining powder and single-crystal X-ray diffraction with SEM/EDX analysis, we demonstrated unambiguously that bimetallic compounds form true solid solutions, rather than simple physical mixtures of the two phases. In these solid solutions, the two metal ions were found to distribute homogeneously throughout the whole crystal, without appreciable enrichment of any of the metals at any region of the crystal (such as tips or rims). Moreover, a careful analysis of the evolution of the cell parameters with the composition of $\mathrm{Co}-\mathrm{Ni}$ and $\mathrm{Co}-\mathrm{Zn}$ bimetallic compounds strongly suggested that $\mathrm{Co}^{2+}$ ions occupy 
preferentially terminal framework positions, leading to well-defined and predictable environments for the metal ions in the framework as a function of the chemical composition of the binary materials. As far as Mn-Ni mixtures is concerned, our data were not conclusive enough to state with certainty whether preferential siting of terminal positions also applied to $\mathrm{Mn}^{2+}$ ions in $\mathrm{Mn}-\mathrm{Ni}$ compounds.

Clear implications of preferential site occupation can be envisaged on the catalytic behavior of the materials, since this should influence, e.g., the average distance between neighbor metal active sites as a function of the chemical composition of the solid solution, or the probability to have metal site isolation. In the following, we will show that this is indeed the case for the aerobic oxidation of cumene.

\section{Catalytic studies}

In order to evaluate the catalytic activity of the materials for the aerobic oxidation of cumene, we first considered the monometallic compounds, Co-BTC, Ni-BTC, Cu-BTC and $\mathrm{Zn}$-BTC. Table 1 summarizes the results obtained, together with a blank (autocatalyzed) reaction measured under the same conditions. While Ni-BTC and Zn-BTC were basically not active under the reaction conditions used, $\mathrm{Cu}$-BTC produced only a slight increase of the CM conversion as compared with the auto-catalyzed process (entries 1 and 4 in Table 1). Conversely, Co-BTC afforded a noticeable 10-fold increase of the CM conversion with respect to the blank experiment (entries 1 and 2). Thus, 49\% conversion of CM was obtained after $7 \mathrm{~h}$ over Co-BTC. Meanwhile, the auto-catalyzed reaction produced only $5 \% \mathrm{CM}$ conversion after the same reaction time. However, the CHP selectivity attained over Co-BTC was only moderate, 69\%, being 2-phenyl-2propanol (PP) the main side product formed (30\% selectivity), along with a small amount (ca. 1\%) of acetophenone (AP) coming from non-selective decomposition side reactions. This is in sharp contrast with the excellent CHP selectivity obtained in the blank experiment (94\%). The reason for the lower selectivity to CHP attained over Co-BTC with respect to the non-catalyzed reaction is that Co-BTC can also catalyze the decomposition of CHP, producing $\mathrm{PP}$ and AP and decreasing the final CHP yield (Scheme 1). The time evolution of products and selectivity to CHP obtained over CoBTC is shown in Fig. 2. 
Table 1. Summary of results of CM oxidation over monometallic MOFs. ${ }^{a}$

\begin{tabular}{clcccc}
\hline Entry & MOF & $\begin{array}{c}\text { Conversion } \\
(\%)\end{array}$ & $\begin{array}{c}\text { Selectvity to } \\
\text { CHP }(\%)\end{array}$ & $\begin{array}{c}\text { Productivity of } \\
\mathrm{CHP}\left(\mathrm{g} \cdot \mathrm{L}^{-1} \cdot \mathrm{h}^{-1}\right)\end{array}$ & E-factor $^{c}$ \\
\hline 1 & Blank & 5 & 94 & 8 & 0.070 \\
2 & Co-BTC & 49 & 69 & 53 & 0.453 \\
3 & Ni-BTC & 6 & 91 & 8 & 0.102 \\
4 & Cu-BTC & 12 & 94 & 18 & 0.057 \\
5 & Zn-BTC & 3 & 95 & 4 & 0.048 \\
\hline
\end{tabular}

${ }^{a}$ Reaction conditions: $1 \mathrm{~mL}$ of cumene $(7.17 \mathrm{mmol})$, catalyst (cumene-to-metal molar ratio $=150), p\left(\mathrm{O}_{2}\right)=4 \mathrm{bar}, 90^{\circ} \mathrm{C}, 7 \mathrm{~h}$ of reaction. ${ }^{b}$ Conversion $(\mathrm{mol} \%)$, determined by GC. ${ }^{c}$ Environmental factor, calculated as the mass ratio of waste to desired product.

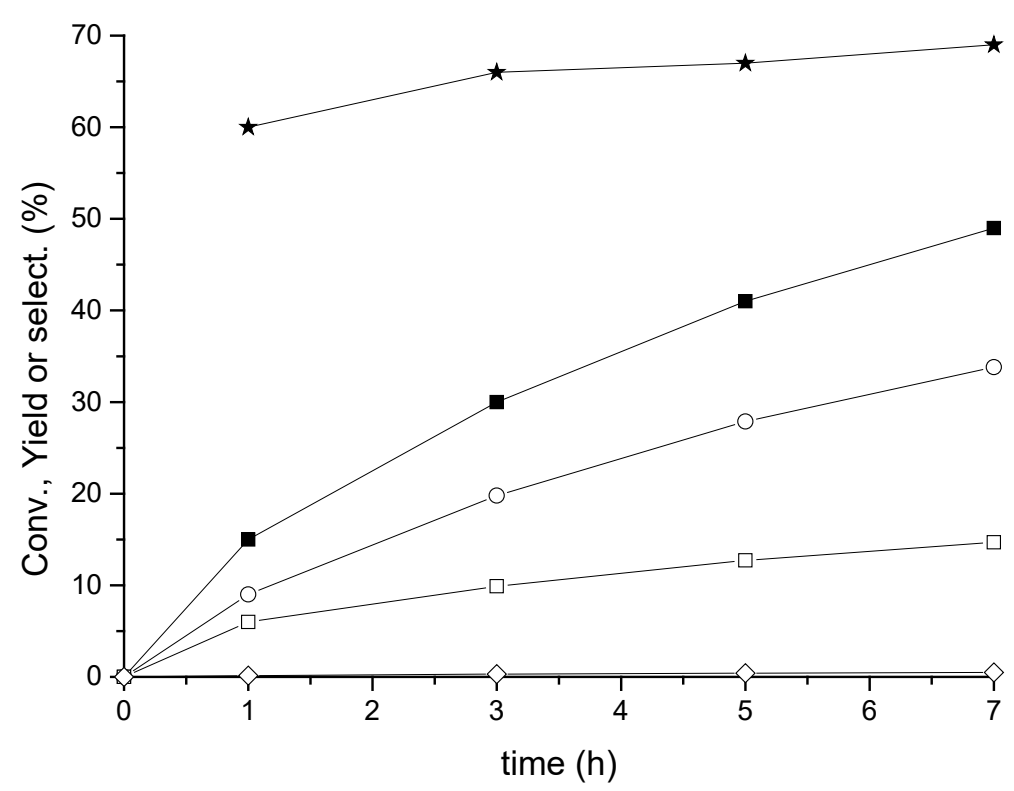

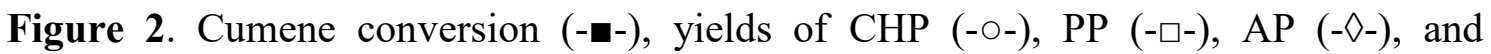
selectivity to CHP (-*-) obtained over Co-BTC. Reaction conditions: $1 \mathrm{~mL}$ of cumene $(7.17 \mathrm{mmol}$ ), catalyst (cumene-to-Co molar ratio $=150), p\left(\mathrm{O}_{2}\right)=4$ bar, $90^{\circ} \mathrm{C}$.

The activity of Co-BTC calculated as turnover frequency (TOF) at short reaction time was $22.5 \mathrm{~h}^{-1}$; i.e., $22.5 \mathrm{mmols}$ of $\mathrm{CM}$ were converted per mmol of metal and per hour. (This TOF value was calculated between 0 and 1 hour of reaction, with a CM conversion below $\sim 15 \%$ ). However, productivity of the reaction can also be expressed in terms of grams of CHP produced per liter of cumene and per hour $\left(\mathrm{g} \mathrm{L}^{-1} \mathrm{~h}^{-1}\right)$. Note that this quantity takes into account the CM conversion over time and CHP selectivity, but it is independent of the amount of metal catalyst used. This makes the comparison between 
catalyzed and auto-catalyzed experiments straightforward. Productivities calculated in this way for catalyzed and auto-catalyzed reactions have also been included in Table 1. Thus, in spite of affording a lower selectivity to CHP, the use of Co-BTC still produces an almost 7-fold productivity increase with respect to the blank experiment: from 8 to 53 $\mathrm{g} \mathrm{L}^{-1} \mathrm{~h}^{-1}$ during the first $7 \mathrm{~h}$ of reaction, respectively (compare entries 1 and 2 in Table 1). However, these results are still far from optimum due to the low CHP selectivity and the generation of relatively large amount of byproducts (viz. PP and AP), resulting in an Efactor $^{34}$ of 0.453 (as compared with 0.070 for the auto-catalyzed process). We then started an optimization of the reaction conditions to improve these results.

\section{Optimization of the reaction conditions}

Reaction temperature. In order to determine the influence of various reaction parameters on the performance of the trimesate MOFs, we first considered the reaction temperature. Fig. 3 summarizes the catalytic results obtained for Co-BTC and autocatalyzed experiments at reaction temperatures ranging from $50^{\circ}$ to $95^{\circ} \mathrm{C}$. Conversions and selectivities reported in this figure correspond to $7 \mathrm{~h}$ of reaction.

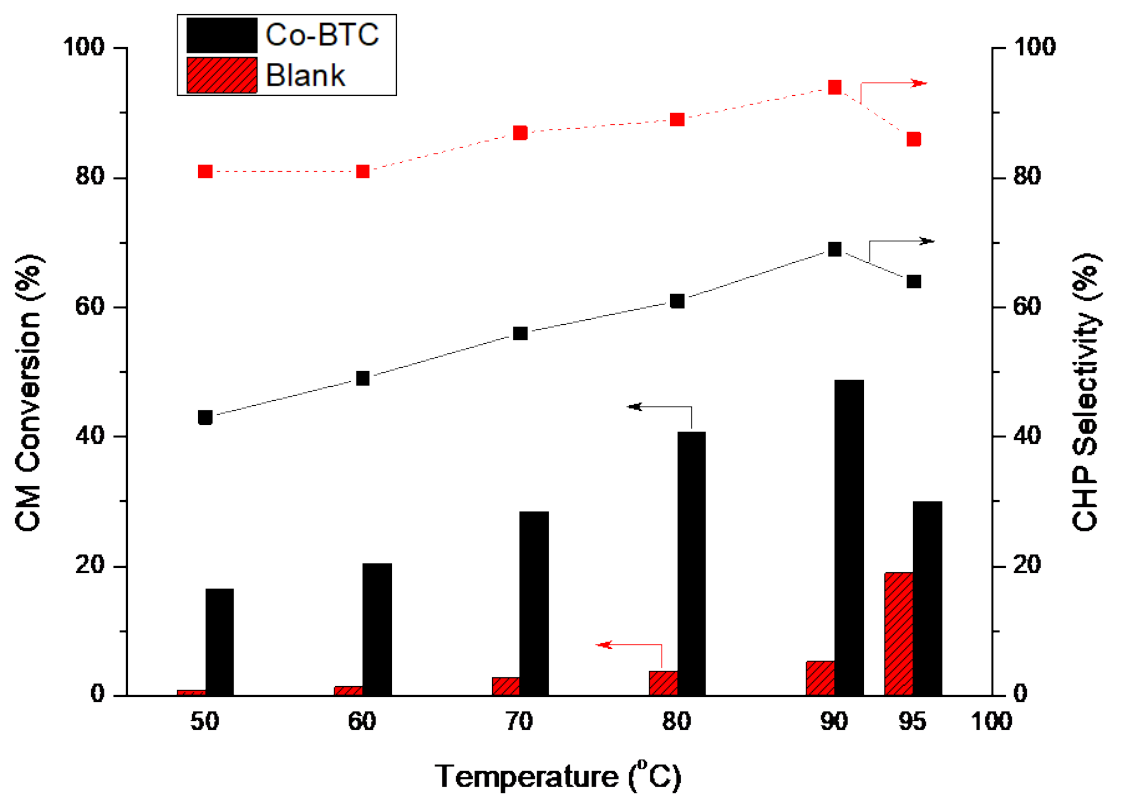

Figure 3. CM conversion (bars) and CHP selectivity (lines) attained over Co-BTC and auto-catalyzed reactions as a function of the reaction temperature.

It is evident from the above results that an increase in the temperature produces a concomitant and highly desired increase of both CM conversion and selectivity to CHP 
for both, MOF catalyzed and auto-catalyzed reactions in the temperature range from $50^{\circ}$ to $90^{\circ} \mathrm{C}$. Unfortunately, increasing further the reaction temperature above $90^{\circ} \mathrm{C}$ produced a gradual dehydration of Co-BTC, which was associated with an evident color change (from pink to purple) and a severe loss of the crystallinity of the MOF (see Fig. S1 in the Supporting Information). Note that water molecules directly coordinated to the metal ions engage in a hydrogen bonding network that holds the MOF structure, so their removal causes the collapse of the framework. This crystallinity loss translated into a severe decrease of the catalytic activity of the material (from $49 \%$ conversion at $90^{\circ} \mathrm{C}$ to $30 \%$ at $95^{\circ} \mathrm{C}$ ) and a slight decrease of selectivity to CHP as well (from $69 \%$ to $64 \%$ ). Therefore, $90^{\circ} \mathrm{C}$ can be considered as the optimum reaction temperature for this type of materials.

Chemical composition: Bimetallic MOFs. Next, we evaluated the catalytic activity of various bimetallic MOFs having different $\mathrm{Co} / \mathrm{Ni}$ ratios. Table 2 summarizes the results obtained after $7 \mathrm{~h}$ at a reaction temperature of $90^{\circ} \mathrm{C}$, while time-conversion plots are shown in Fig. S2 in the Supporting Information).

Table 2. Summary of results of CM oxidation over mono- and bimetallic Co-Ni MOFs of various compositions. ${ }^{a}$

\begin{tabular}{clccccc}
\hline Entry & MOF & $\begin{array}{c}\mathrm{CM}: \mathrm{Co}^{2+} \\
\text { ratio }\end{array}$ & $\begin{array}{c}\text { Conv. } \\
(\%)\end{array}$ & $\begin{array}{c}\text { Select. } \\
\mathrm{CHP}(\%)\end{array}$ & $\begin{array}{c}\text { Productivity } \\
\mathrm{CHP}\left(\mathrm{g} \cdot \mathrm{L}^{-1} \cdot \mathrm{h}^{-1}\right)\end{array}$ & E-factor \\
\hline 1 & Blank & - & 5 & 94 & 8 & 0.070 \\
2 & Co-BTC & 150 & 49 & 69 & 53 & 0.453 \\
3 & Co33Ni-BTC & 450 & 35 & 76 & 41 & 0.323 \\
4 & Co5Ni-BTC & 3000 & 30 & 91 & 43 & 0.098 \\
5 & Co2Ni-BTC & 7500 & 19 & 91 & 27 & 0.100 \\
6 & Co1Ni-BTC & 15000 & 14 & 92 & 20 & 0.089 \\
7 & Ni-BTC & - & 6 & 91 & 8 & 0.102 \\
\hline${ }^{a}$ Reaction conditions: 1 & $\mathrm{~mL}$ of cumene $(7.17 \mathrm{mmol})$, catalyst $(14 \mathrm{mg}$ of MOF, the \\
resulting cumene to $\mathrm{Co}^{2+}$ molar ratio is indicated $), p\left(\mathrm{O}_{2}\right)=4$ bar, $90^{\circ} \mathrm{C}, 7 \mathrm{~h}$ of reaction.
\end{tabular}

As it can be seen in Table 2 and Fig. S2, there is a progressive and gradual increase of $\mathrm{CM}$ conversion with the Co content of the MOF in the bimetallic compound, on passing from the pure Ni-BTC (6\%) to pure Co-BTC (49\%). Meanwhile, the selectivity to CHP is maintained above $90 \%$ for a Co content below $5 \%$, and then starts to decrease rapidly as the Co content increases further. Therefore, the Co5Ni-BTC bimetallic compound (containing $5 \mathrm{~mol} \%$ of $\mathrm{Co}$ ) was considered to be the best compromise between $\mathrm{CM}$ conversion and CHP productivity and selectivity. With respect to the auto-catalyzed 
reaction, Co5Ni-BTC provides a much higher productivity, 43 vs. $8 \mathrm{~g} \mathrm{~L}^{-1} \mathrm{~h}^{-1}$, while still maintaining an excellent selectivity to CHP (91\% CHP vs 94\%). Accordingly, the Efactor obtained with this bimetallic catalyst is low (0.098) and not far from that of the auto-catalyzed process (0.070).

The gradual slowdown of the oxidation reaction on moving from $\mathrm{Co}-\mathrm{BTC}$ to $\mathrm{Ni}$ BTC, and all the bimetallic compounds in between, can be attributed to a dilution effect, since the total amount of $\mathrm{Co}^{2+}$ ions in the solid is progressively reduced (from a $\mathrm{CM}: \mathrm{Co}^{2+}$ ratio of 150 in pure $\mathrm{Co}-\mathrm{BTC}$ down to 15000 in $\mathrm{Co} 1 \mathrm{Ni}-\mathrm{BTC})$. Note that $\mathrm{Ni}^{2+}$ ions are basically inactive for this reaction (compare entries 1 and 6 in Table 2). In turn, $\mathrm{Co}^{2+}$ ions also catalyze side reactions leading to CHP decomposition (again $\mathrm{Ni}^{2+}$ ions are inactive for CHP decomposition, see Fig. S3 in the Supporting Information for the corresponding CHP decomposition experiments), so this can also explain the observed progressive decrease of CHP selectivity as the cobalt content of the solid increases.

Nevertheless, we wanted to explore in more detail if this "dilution effect" was the only reason for the excellent CHP selectivity attained over our best catalyst, the Co5NiBTC sample. To address this point, we determined the catalytic properties of a physical mixture of pure Ni-BTC and Co-BTC monometallic compounds in the appropriate ratio to obtain a $\mathrm{Co}: \mathrm{Ni}$ molar ratio of 5:95; i.e., the same $\mathrm{Co}$ : Ni ratio found in bimetallic Co5NiBTC, and keeping a $\mathrm{CM}: \mathrm{Co}^{2+}$ ratio of 3000 . This physical mixture produced a similar level of CM conversion (28\%) as the bimetallic MOF (30\%) after $7 \mathrm{~h}$ of reaction, with a CHP selectivity of $85 \%$ (see Table 3 ). However, although this CHP selectivity was much higher than that of pure Co-MOF (i.e., 69\%), it was still significantly below the selectivity obtained for the Co5Ni-BTC bimetallic compounds at the same level of conversion ( $85 \%$ vs. 91\%). The differences between Co5Ni-BTC and the physical mixture are even higher if we analyze the data corresponding to lower CM conversion and shorter reaction time, as it is clearly seen in the conversion-selectivity plot shown in Fig S4 in the Supporting Information. Therefore, although the physical dilution effect probably plays an important role, it is probably not the only effect determining the excellent CHP selectively attained over Co5Ni-BTC. 
Table 3. Catalytic results obtained for the $\mathrm{CM}$ oxidation over bimetallic Co5Ni-BTC and a physical mixture of pure Co-BTC and Ni-BTC in a 5:95 ratio. The results obtained with pure Co-BTC are also included for comparison. ${ }^{a}$

\begin{tabular}{clccc}
\hline Entry & MOF & $\begin{array}{c}\text { Conv. } \\
(\%)\end{array}$ & $\begin{array}{c}\text { Select. } \\
\text { CHP }(\%)\end{array}$ & $\begin{array}{c}\text { Productivity } \\
\mathrm{CHP}\left(\mathrm{g} \cdot \mathrm{L}^{-1} \cdot \mathrm{h}^{-1}\right)\end{array}$ \\
\hline 1 & Co-BTC & 49 & 69 & 53 \\
2 & Co5Ni-BTC & 30 & 91 & 43 \\
3 & Co-BTC + Ni-BTC & 28 & 85 & 38 \\
\hline
\end{tabular}

${ }^{a}$ Reaction conditions: $1 \mathrm{~mL}$ of cumene $(7.17 \mathrm{mmol})$, catalyst $(\mathrm{Co}: \mathrm{Ni}=5: 95$ and $\mathrm{CM}$ to $\mathrm{Co}^{2+}$ molar ratio $\left.=3000\right), p\left(\mathrm{O}_{2}\right)=4$ bar, $90^{\circ} \mathrm{C}, 7 \mathrm{~h}$ of reaction. ${ }^{b}$ Same conditions as in Table 1 and 2; i.e., $\mathrm{CM}$ to $\left.\mathrm{Co}^{2+}=150\right)$.

As we concluded in our previous work, ${ }^{32} \mathrm{Co}^{2+}$ ions in the bimetallic MOFs distribute homogeneously throughout the whole crystalline framework, with a high tendency to occupy terminal positions in the first place (up to a $33 \%$ concentration). It is then reasonable to assume that at such a low concentration ( $5 \%$ total cobalt content), most of the $\mathrm{Co}^{2+}$ ions will occupy terminal positions.

In the M-BTC crystalline framework, each ion in a terminal site is surrounded by 8 closest neighbors: 6 bridging atoms at distances $d_{\text {term-bridg }}=5.89 \AA$ (two sites), $5.63 \AA$ (two sites) and $5.60 \AA$ (two sites), and 2 terminal atoms at distances $d_{\text {term-term }}=6.53 \AA$. Beyond these 8 closest neighbors, other metal-metal contacts are too distant (e.g., $d_{\text {term- }}$ term $=16.11 \AA, d_{\text {term-bridg }}=10.40 \AA$ and $d_{\text {bridg-bridg }}=8.32 \AA$ ), so that they can be neglected. Taking into account this spatial distribution of metal sites and the preferred site occupation of terminal sites by $\mathrm{Co}^{2+}$ ions, the arrangement of $\mathrm{Co}^{2+}$ ions in the structure can be schematically represented as shown in Fig. 4. Then, the statistical probability of each configuration can be calculated as a function of the total cobalt content of the bimetallic compound (further details on the calculation procedure are given in the Supporting Information). 

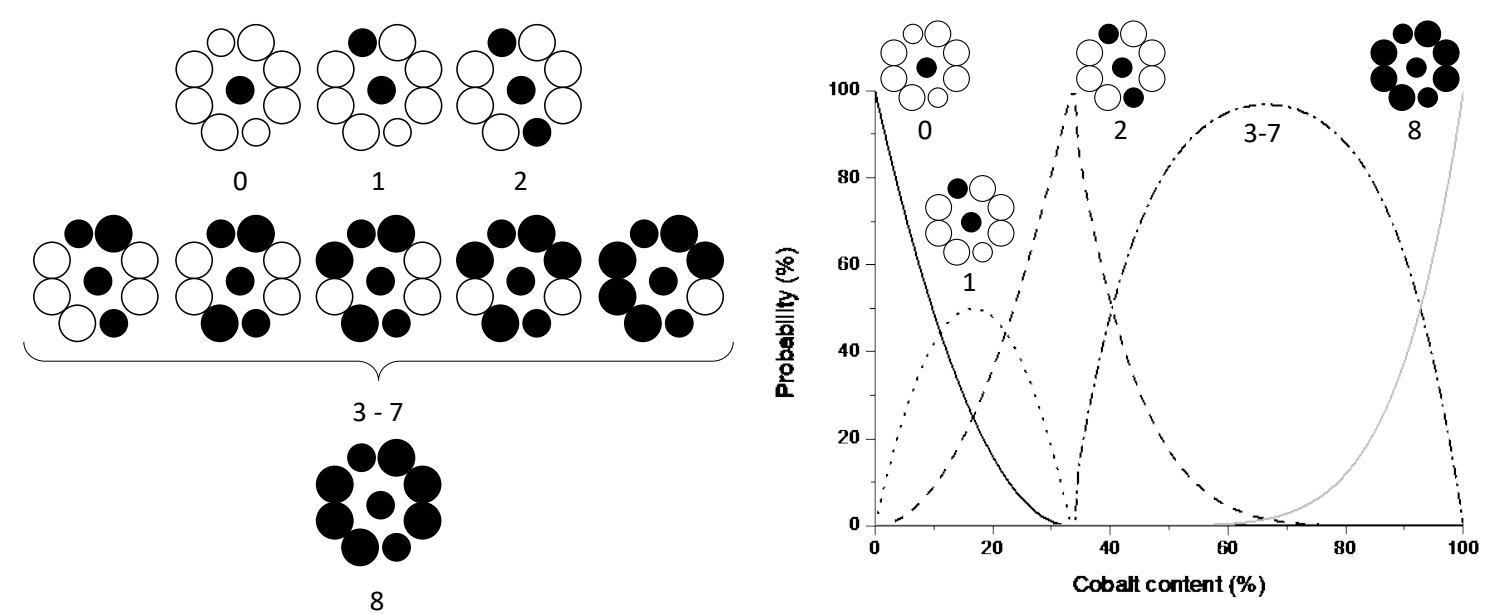

Figure 4. (Left) Possible arrangement of $\mathrm{Co}^{2+}$ ions in CoxNi-BTC bimetallic compounds. In these schematic drawings, a central $\mathrm{Co}^{2+}$ ion (black) in a terminal position is surrounded by 8 closest neighbors (less than $7 \AA$ apart): 6 bridging sites (large circles) and 2 terminal sites (small circles), that can be occupied either by another $\mathrm{Co}^{2+}$ ion (black) or a $\mathrm{Ni}^{2+}$ ion (white). The number of $\mathrm{Co}^{2+}$ closest neighbors in each arrangement is indicated below each picture. (Right) Statistical probability of having each of the $\mathrm{Co}^{2+}$ arrangements as a function of the total cobalt content in the bimetallic compound, calculated as detailed in the Supporting Information.

Note that, according to the simple model considered above, the probability of having an isolated $\mathrm{Co}^{2+}$ site (i.e., a $\mathrm{Co}^{2+}$ ion surrounded by $8 \mathrm{Ni}^{2+}$ ions) falls sharply at relatively low cobalt concentration. Thus, the probability of having site isolation would be of around $73 \%$ in Co5Ni-BTC, while this probability would fall down to $15 \%$ for Co20Ni-BTC, and to zero for Co33Ni-BTC, in which all $\mathrm{Co}^{2+}$ would be surrounded by two other $\mathrm{Co}^{2+}$ ions. This means that statistically $73 \%$ of the total $\mathrm{Co}^{2+}$ sites in Co5NiBTC will have no other $\mathrm{Co}^{2+}$ atom in any of the 8 closest neighbor positions. Or, in other words, that two neighbor $\mathrm{Co}^{2+}$ ions will be at least 8-9 $\AA$ apart.

It is well known that site isolation can be crucial in determining the observed catalytic properties of a material. ${ }^{35-37}$ This is also the present case for the aerobic oxidation of cumene. Once a CHP molecule is formed in the main reaction, it can either desorb into the liquid phase, or it can undergo secondary oxidation or decomposition events at the surface of the catalyst that will consume CHP (decreasing the final CHP selectivity of the overall catalytic process). It is evident that if the average separation between two neighbor $\mathrm{Co}^{2+}$ sites in the bimetallic compound is large due to site isolation, CHP decomposition and other secondary oxidation events are less likely to take place, and this will result in an overall higher CHP selectivity of the catalytic process, as show in Scheme 2. Note that the beneficial effects of site isolation on the catalytic properties of the bimetallic 
compounds are only possible because $\mathrm{Ni}^{2+}$ are inactive in CHP decomposition. Thus, from a practical point of view, our catalysts can be viewed as an array of $\mathrm{Co}^{2+}$ active sites diluted in an inert Ni-BTC matrix.
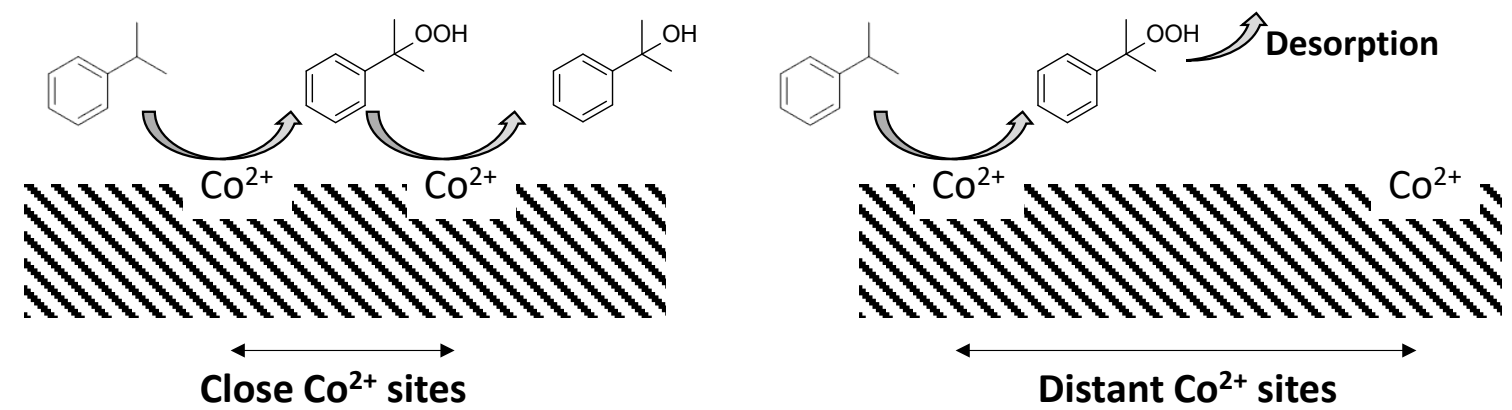

Scheme 2. CHP decomposition is more likely to occur on close $\mathrm{Co}^{2+}$ sites (left part) than on distant, site isolated $\mathrm{Co}^{2+}$ sites (right part).

To lend further support to this "site isolation" or "matrix isolation" effect as the responsible for the high CHP selectivities observed, we have extended our results to another series of bimetallic Mn-Ni compounds, in which the catalytically active sites $\left(\mathrm{Mn}^{2+}\right)$ are likewise diluted in an inert $\mathrm{Ni}^{2+}$ matrix. As we described in our previous report, ${ }^{32}$ we were not able to prepare pure Mn-BTC with the same crystalline structure as the Co- and Ni-BTC compounds. Nevertheless, we were able to prepare isoreticular MnNi bimetallic MOFs up to a maximum Mn concentration of $50 \%$.

We thus prepared Mn-Ni bimetallic trimesates having 1\%, 2\% and 5\% Mn and used them as catalysts for the aerobic oxidation of $\mathrm{CM}$. The catalytic results obtained are summarized in Table 4, which includes also for comparison the results of a physical mixture of pure Mn-BTC and Ni-BTC in a Mn:Ni molar ratio of 2:98.

Table 4. Summary of results of CM oxidation over mono- and bimetallic Mn-Ni MOFs of various compositions, and of a physical mixture (Mn-BTC+Ni-BTC) in a 2:98 molar ratio. $^{a}$

\begin{tabular}{clccccc}
\hline Entry & MOF & $\begin{array}{c}\text { CM:Mn } \\
\text { ratio }\end{array}$ & $\begin{array}{c}\text { Conv. } \\
(\%)\end{array}$ & $\begin{array}{c}\text { Select. } \\
\text { CHP }(\%)\end{array}$ & $\begin{array}{c}\text { Produc. CHP } \\
\left(\mathrm{g} \cdot \mathrm{L}^{-1} \cdot \mathrm{h}^{-1}\right)\end{array}$ & E-factor \\
\hline 1 & Blank & - & 5 & 94 & 8 & 0.070 \\
2 & Mn5Ni-BTC & 3000 & 43 & 77 & 51 & 0.307 \\
3 & Mn2Ni-BTC & 7500 & 30 & 87 & 41 & 0.156 \\
4 & Mn1Ni-BTC & 15000 & 25 & 90 & 36 & 0.112 \\
5 & Mn-BTC + Ni-BTC & 7500 & 32 & 82 & 42 & 0.222 \\
\hline
\end{tabular}

${ }^{a}$ Reaction conditions: $1 \mathrm{~mL}$ of cumene $(7.17 \mathrm{mmol})$, catalyst $(14 \mathrm{mg}$ of MOF, the resulting cumene to $\mathrm{Mn}^{2+}$ molar ratio is indicated), $p\left(\mathrm{O}_{2}\right)=4$ bar, $90^{\circ} \mathrm{C}, 7 \mathrm{~h}$ of reaction. 
As compared with the Co-Ni compounds, the Mn-Ni MOFs were slightly more active for CM conversion, but less selective to CHP (compare data in Tables 2 and 4). Nevertheless, the same general trends are also observed throughout the series of bimetallic compounds: an increase of the $\mathrm{Mn}$ :Ni ratio in the solid solution produces a gradual increase in the CM conversion and a decrease of the selectivity to CHP. Again, the solid solution compounds provide a higher CHP selectivity than simple physical mixtures: $87 \%$ vs. $82 \%$ (compare entries 3 and 5 in Table 4). In our opinion, these finding support the hypothesis of the site isolation effect as one of the reasons behind the elevated CHP selectivities obtained with bimetallic trimesate MOFs.

Stability and reusability of the MOFs. When dealing with any process in heterogeneous catalysis, stability and recyclability of the catalyst become relevant aspects. And particularly when considering the sustainability of the overall process. According to the $\mathrm{XRD}$ of the materials recovered after the reaction, all $\mathrm{Co}-\mathrm{Ni}$ and $\mathrm{Mn}-\mathrm{Ni}$ bimetallic compounds described above were found to maintain their crystallinity (see Fig. S2 in Supporting Information). Accordingly, the materials were found to maintain the catalytic activity and CHP selectivity practically unchanged for at least 5 consecutive catalytic cycles, as shown in Fig. 5 for sample Co5Ni-BTC (see also Fig. S6 in Supporting Information for sample Mn2Ni-BTC). Moreover, analysis of the liquid filtrate after the reaction by ICP-OES does not revealed the presence of significant amounts of metals in solution $\left(\mathrm{Co}^{2+}, \mathrm{Ni}^{2+}\right.$ or $\left.\mathrm{Mn}^{2+}\right)$, which indicates that leaching of the metal ions from the MOF to the liquid medium is very limited. 


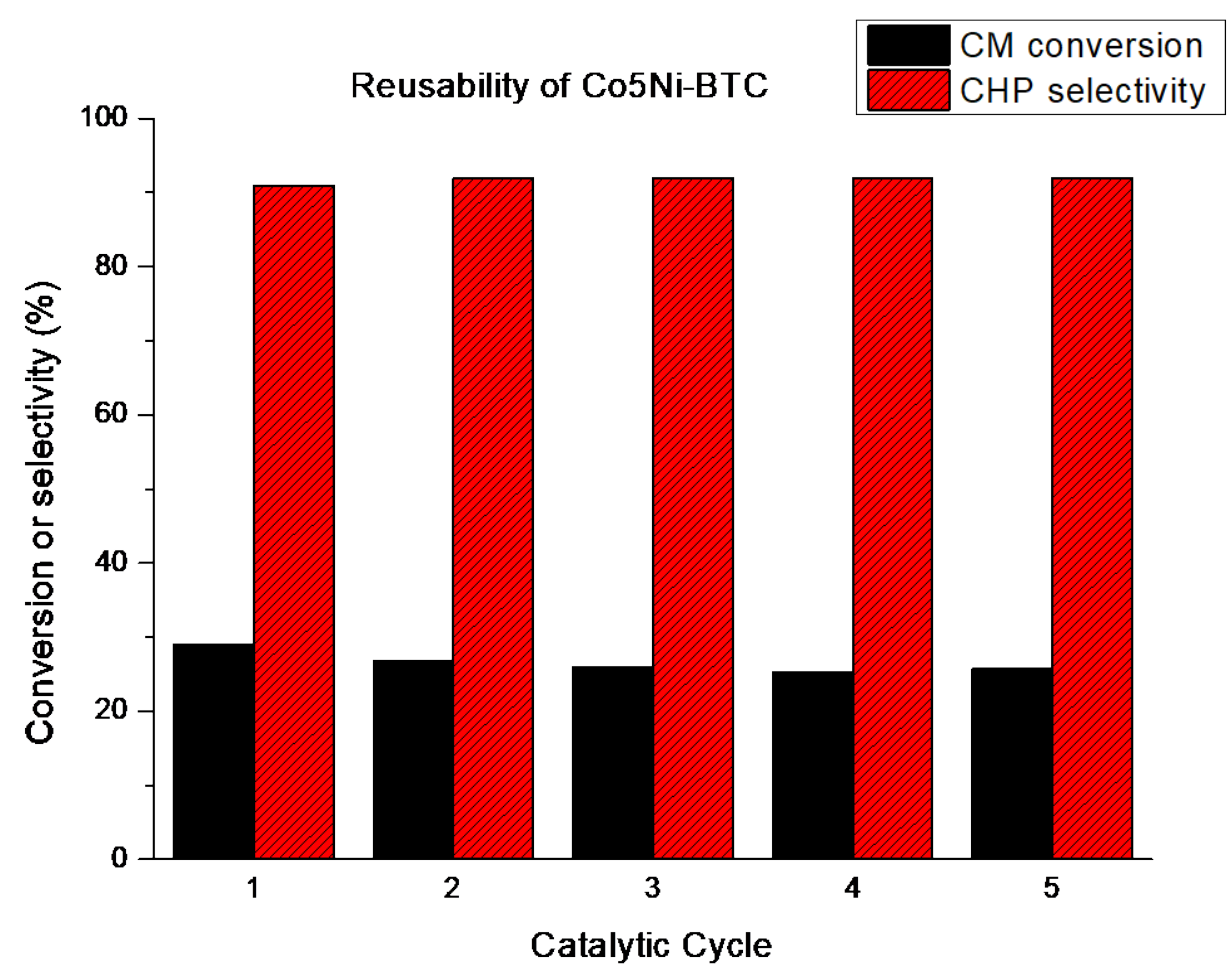

Figure 5. Reusability of the Co5Ni-BTC bimetallic compound in five consecutive catalytic cycles.

\section{Conclusions}

We have shown that bimetallic Co-Ni and Mn-Ni trimesate MOFs prepared by a fast aqueous synthesis are excellent and reusable catalysts for the selective oxidation of cumene to cumene hydroperoxide. Isolation of $\mathrm{Co}^{2+}\left(\right.$ or $\left.\mathrm{Mn}^{2+}\right)$ in an inert Ni-BTC framework by progressively decreasing the total cobalt content of the binary solid solution is a good strategy to optimize CHP selectivity above $90 \%$, and to minimize production of side products (mainly PP). This beneficial effect is probably due in part to a dilution effect: since $\mathrm{Co}^{2+}$ are the only sites that can catalyze CHP decomposition, we can expect a drop of the CHP selectivity as the cobalt content increases. Meanwhile, the homogeneous distribution of $\mathrm{Co}^{2+}$ ions throughout the Ni-BTC framework and their preferential occupation of terminal sites allowed us to construct a simple model to calculate the statistical probability of having isolated $\mathrm{Co}^{2+}$ sites, surrounded only by (inactive) $\mathrm{Ni}^{2+}$ sites in the closest positions, as a function of the total cobalt content of the bimetallic compound. In this way, the excellent CHP selectivity (91\%) obtained over our best compound, Co5Ni-BTC, can be explained in terms of a "site isolation" or "matrix isolation" effect. Indeed, according to our model, this sample statistically contains $73 \%$ of the total $\mathrm{Co}^{2+}$ ions as isolated sites, so that CHP decomposition/over-oxidation 
processes at the surface of the catalyst are not likely to occur before CHP desorption takes place. This "site isolation" effect was further supported by similar findings on $\mathrm{Mn}-\mathrm{Ni}$ bimetallic compounds.

\section{Supporting Information}

$\mathrm{XRD}$ of pristine and dehydrated Co-BTC; temporal evolution of products over Co-Ni bimetallic compounds; CHP decomposition experiments; conversion-selectivity plots of Co5Ni-BTC and Co-BTC/Ni-BTC physical mixture; XRD of Co5Ni-BTC and Mn2NiBTC after 5 reuses; reusability of Mn2Ni-BTC; calculation of probabilities of different $\mathrm{Co}^{2+}$ arrangements

\section{ACKNOWLEDGEMENTS:}

This project has received funding from the European Union's Horizon 2020 research and innovation programme under the Marie Sklodowska-Curie grant agreement No. 641887 (project acronym: DEFNET) and the Spanish Government through projects MAT201782288-C2-1-P and Severo Ochoa (SEV-2016-0683).

\section{References}

1. Zakoshansky, V. M., The Cumene Process for Phenol-Acetone Production. Petrol. Chem. 2007, 47, 301-313, DOI 10.1134/S0965544107040135.

2. Reddy, P. V. L.; Kim, K. H.; Kavitha, B.; Kumar, V.; Raza, N.; Kalagara, S., Photocatalytic degradation of bisphenol A in aqueous media: A review. J. Environ. Manage. 2018, 213, 189205, DOI 10.1016/j.jenvman.2018.02.059.

3. Tsuji, J.; Yamamoto, J.; Corma, A.; Rey, F., Method for producing propylene oxide 1998, US Patent, 6211388,

4. Tsuji, J.; Yamamoto, J.; Ishino, M.; Oku, N., Development of new propylene oxide process. Sumitomo Kagaku 2006, 1, 4-10,

5. Sheldon, R. A.; Arends, I. W. C. E., Catalytic oxidations mediated by metal ions and nitroxyl radicals. J. Mol. Catal. A-Chem. 2006, 251, 200-214, DOI 10.1016/j.molcata.2006.02.016. 6. Kasperczyk, K.; Orlińska, B.; Zawadiak, J., Aerobic oxidation of cumene catalysed by 4alkyloxycarbonyl-N-hydroxyphthalimide. Cent. Eur. J. Chem. 2014, 12, 1176-1182, DOI 10.2478/s11532-014-0565-8.

7. Melone, L.; Prosperini, S.; Ercole, G.; Pastoria, N.; Punta, C., Is it possible to implement $\mathrm{N}$-hydroxyphthalimide homogeneous catalysis for industrial applications? A case study of cumene aerobic oxidation. J. Chem. Technol. Biotechnol. 2014, 89, 1370-1378, DOI $10.1002 / j c t b .4213$.

8. Sapunov, V. N.; Kurganova, E. A.; Koshel, G. N., Kinetics and Mechanism of Cumene Oxidation Initiated by N-Hydroxyphthalimide. Int. J. Chem. Kinet. 2018, 50, 3-14, DOI 10.1002/kin.21135. 
9. Hou, H. Y.; Shu, C. M.; Tsai, T. L., Reactions of cumene hydroperoxide mixed with sodium hydroxide. J. Hazard. Mater. 2008, 152, 1214-1219, DOI 10.1016/j.jhazmat.2007.07.118.

10. Armstrong, G. P.; Hall, R. H.; Quin, D. C.; Turck, H. W., Manufacture of peroxidic compounds 1965, US Patent, 3187055,

11. Zakoshansky, V. M.; Griaznov, K.; Vasilieva, I. I.; Fulmer, J. W.; Kight, W. D., Cumene oxidation process 1998, US Patent 5767322,

12. Feder, R. L.; Fuhrmann, R.; Pisanchyn, J.; Elishewitz, S.; Insinger, T. H.; Mathew, C. T., Continuous process for preparing cumene hydroperoxide 1975, US Patent 3907901,

13. Srivastava, R. K.; Srivastava, R. D., Kinetics of liquid phase oxidation of cumene with $\mathrm{Cr} 2 \mathrm{O} 3, \mathrm{MnO} 2$ and $\mathrm{Fe} 2 \mathrm{O} 3$ catalysts. J. Catal. 1975, 39, 317-323, DOI 10.1016/00219517(75)90297-3.

14. Hsu, Y. F.; Yen, M. H.; Cheng, C. P., Autooxidation of cumene catalyzed by transition metal compounds on polymeric supports. J. Mol. Catal. A-Chem. 1996, 105, 137-144, DOI 10.1016/1381-1169(95)00205-7.

15. Maksimov, Y. V.; Suzdalev, I. P.; Tsodikov, M. V.; Kugel, V. Y.; Bukhtenko, O. V.; Slivinsky, E. V.; Navio, J. A., Study of cumene oxidation over zirconia-, titania- and alumina-based complex oxides obtained by sol-gel methods: activity-structure relationships. J. Mol. Catal. A-Chem. 1996, 105, 167-173, DOI 10.1016/1381-1169(95)00188-3.

16. Sheldon, R. A.; Wallau, M.; Arends, I. W. C. E.; Schuchardt, U., Heterogeneous Catalysts for Liquid-Phase Oxidations: Philosophers' Stones or Trojan Horses? Acc. Chem. Res. 1998, 31, 485-493, DOI 10.1021/ar9700163.

17. Matsui, S.; Fujita, T., New cumene-oxidation systems $\mathrm{O} 2$ activator effects and radical stabilizer effects. Catal. Today 2001, 71, 145-152, DOI 10.1016/S0920-5861(01)00450-3.

18. Zhang, M.; Wang, L.; Ji, H.; Wu, B.; Zeng, X., Cumene Liquid Oxidation to Cumene Hydroperoxide over CuO Nanoparticle with Molecular Oxygen under Mild Condition. J. Nat. Gas Chem. 2007, 16, 393-398, DOI 10.1016/S1003-9953(08)60010-9.

19. Yang, W. J.; Guo, C. C.; Tao, N. Y.; Cao, J., Aerobic Oxidation of Cumene to Cumene Hydroperoxide Catalyzed by Metalloporphyrins. Kinet. Catal. 2010, 51, 194-199, DOI $10.1134 /$ S0023158410020047.

20. Kobotaeva, N. S.; Skorokhodova, T. S.; Ryabova, N. W., Catalytic Systems of Cumene Oxidation Based on Multiwalled Carbon Nanotubes. Russ. J. Phys. Chem. A 2015, 89, 462-468, DOI 10.1134/S0036024415030164.

21. Ghanbari, B.; Ferdosi, S. R.; Tafazolian, H., Solvent-free oxidation of cumene by molecular oxygen catalyzed by cobalt salen-type complexes. Res. Chem. Intermed. 2018, 38, 871-883, DOI 10.1007/s11164-011-0425-5.

22. Llabrés i Xamena, F. X.; Casanova, O.; Galiasso Tailleur, R.; Garcia, H.; Corma, A., Metal organic frameworks (MOFs) as catalysts: A combination of $\mathrm{Cu} 2+$ and $\mathrm{Co} 2+\mathrm{MOFs}$ as an efficient catalyst for tetralin oxidation. J. Catal. 2008, 255, 220-227, DOI 10.1016/j.jcat.2008.02.011.

23. Dhakshinamoorthy, A.; Alvaro, M.; Garcia, H., Atmospheric-Pressure, Liquid-Phase, Selective Aerobic Oxidation of Alkanes Catalysed by Metal-Organic Frameworks. Chem. Eur. J 2011, 17, 6256-6262, DOI 10.1002/chem.201002664.

24. Santiago-Portillo, A.; Navalón, S.; Cirujano, F. G.; Llabrés i Xamena, F. X.; Alvaro, M.; Garcia, H., MIL-101 as Reusable Solid Catalyst for Autoxidation of Benzylic 2 Hydrocarbons in the Absence of Additional Oxidizing Reagent. ACS Catal. 2015, 5, 3216-3224, DOI 10.1021/acscatal.5b00411.

25. Dhakshinamoorthy, A.; Asiri, A. M.; Herance, J. R.; Garcia, H., Metal organic frameworks as solid promoters for aerobicautoxidations. Catal. Today 2018, 306, 2-8, DOI 10.1016/j.cattod.2017.01.018.

26. Kockrick, E.; Lescouet, T.; Kudrik, E. V.; Sorokin, A. B.; Farrusseng, D., Synergistic effects of encapsulated phthalocyanine complexes in MIL-101 for the selective aerobic oxidation of tetralin. Chem. Commun. 2011, 47 (5), 1562-1564, DOI 10.1039/COCC04431H. 
27. Wang, F.; Jia, S.; Li, D.; Yu, B.; Zhang, L.; Liu, Y.; Han, X.; Zhang, R.; Wu, S., Self-template synthesis of $\mathrm{CuO} @ \mathrm{Cu} 3(\mathrm{BTC}) 2$ composite and its application in cumene oxidation. Mater. Lett. 2016, 164, 72-75, DOI 10.1016/j.matlet.2015.09.044.

28. Reinsch, H., "Green" Synthesis of Metal-Organic Frameworks. Eur. J. Inorg. Chem. 2016, 2016, 4290-4299, DOI 10.1002/ejic.201600286.

29. Reinsch, H.; Bueken, B.; Vermoortele, F.; Stassen, I.; Lieb, A.; Lillerud, K. P.; de Vos, D. E., Green synthesis of zirconium-MOFs. CrystEngComm 2015, 17, 4070-4074, DOI 10.1039/c5ce00618j.

30. Julien, P. A.; Mottillo, C.; Friščić, T., Metal-organic frameworks meet scalable and sustainable synthesis. Green Chem. 2017, 19, 2729-2747, DOI 10.1039/c7gc01078h.

31. Gaab, M.; Trukhan, N.; Maurer, S.; Gummaraju, R.; Müller, U., The progression of Albased metal-organic frameworks - From academic research to industrial production and applications. Microporous Mesoporous Mater. 2012, 157, 131-136, DOI 10.1016/j.micromeso.2011.08.016.

32. Nowacka, A.; Briantais, P.; Prestipino, C.; Llabrés i Xamena, F. X., Facile "green" aqueous synthesis of mono- and bimetallic trimesate metal-organic frameworks. ACS Sust. Chem. Eng. 2018, submited for publication.,

33. Yaghi, O. M.; Li, H.; Groy, T. L., Construction of Porous Solids from Hydrogen-Bonded Metal Complexes of 1,3,5-Benzenetricarboxylic Acid. J. Am. Chem. Soc. 1996, 118, 9096-9101, DOI 10.1021/ja960746q.

34. Sheldon, R. A., The E Factor: fifteen years on. Green Chem. 2007, 9, 1261-1384, DOI 10.1039/b713736m.

35. Grasselli, R. K., Site isolation and phase cooperation: Two important concepts in selective oxidation catalysis: A retrospective. Catal. Today 2014, 238, 10-27, DOI 10.1016/j.cattod.2014.05.036.

36. Rogge, S. M. J.; Bavykina, A.; Hajek, J.; Garcia, H.; Olivos-Suarez, A. I.; SepúlvedaEscribano, A.; Vimont, A.; Clet, G.; Bazin, P.; Kapteijn, F.; Daturi, M.; Ramos Fernandez, E. V.; Llabrés i Xamena, F. X.; Van Speybroeck, V.; Gascon, J., Metal-organic and covalent organic frameworks as single-site catalysts. Chem. Soc. Rev. 2017, 46, 3134-3184, DOI 10.1039/c7cs00033b.

37. Osadchii, D. Y.; Olivos-Suarez, A. I.; Szécsényi, A.; Li, G.; Nasalevich, M. A.; Dugulan, I. A.; Serra Crespo, P.; Hensen, E. J. M.; Veber, S. L.; Fedin, M. V.; Sankar, G.; Pidko, E. A.; Gascon, J., Isolated Fe Sites in Metal Organic Frameworks Catalyze the Direct Conversion of Methane to Methanol. ACS Catal. 2018, 8, 5542-5548, DOI 10.1021/acscatal.8b00505. 


\section{TOC/Abstract graphic}

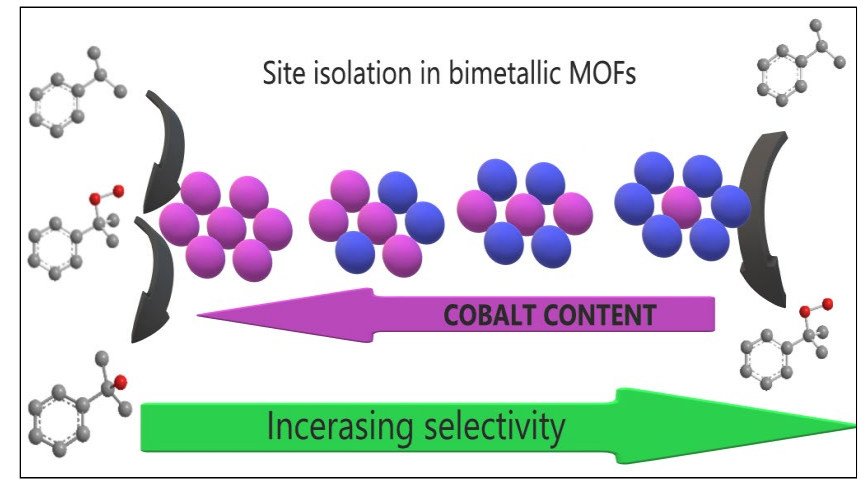

For Table of Contents Use Only

\section{Synopsis}

Effective site isolation ("matrix isolation effect") in bimetallic MOFs leads to highly selective and reusable catalysts for the aerobic oxidation of cumene to cumene hydroperoxide. 